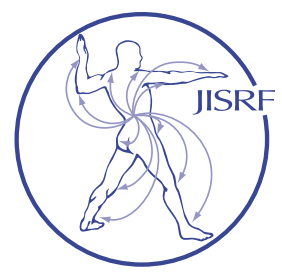

\title{
An Overview of Trauma Center Levels and Disparities in Rural Trauma Care
}

Dave, U'; Gosine, $B^{2}$; Palaniappan, $A^{3}$

\section{Abstact}

Trauma centers in the United States focus on providing care to patients who have suffered injuries and may require critical care. These trauma centers are classified into five different levels: Level I to Level V. Level V trauma centers are the least comprehensive, providing minimal 24hour care and resuscitation, and Level I trauma centers are the most comprehensive, accepting the most severely injured patients and always delivering care through the use of an attending surgeon. However, there is a major inequity in access to trauma centers across the United States, especially amongst rural residents. Level III to Level V trauma centers tend to be dominantly situated in rural and underserved areas. Furthermore, trauma centers tend to be widely dispersed with respect to rural areas. Therefore, these areas tend to have a greater mortality rate in relation to traumatic injuries. Improvements in access to hightier traumatic care must occur in order to reduce mortality due to traumatic injuries in underserved rural areas. Possible improvements to rural trauma care include bolstering the quality of care in Level III trauma centers, increasing Level II center efficiency through the involvement of orthopedic traumatologists, placing medical helicopter bases in more strategic locations that enable transport teams to reach other trauma centers faster, building more Level I and Level II trauma centers, and converting Level III centers into either Level I or Level II centers.

Keywords: trauma centers, rural access, disparities, orthopedics, traumatologists, surgeons

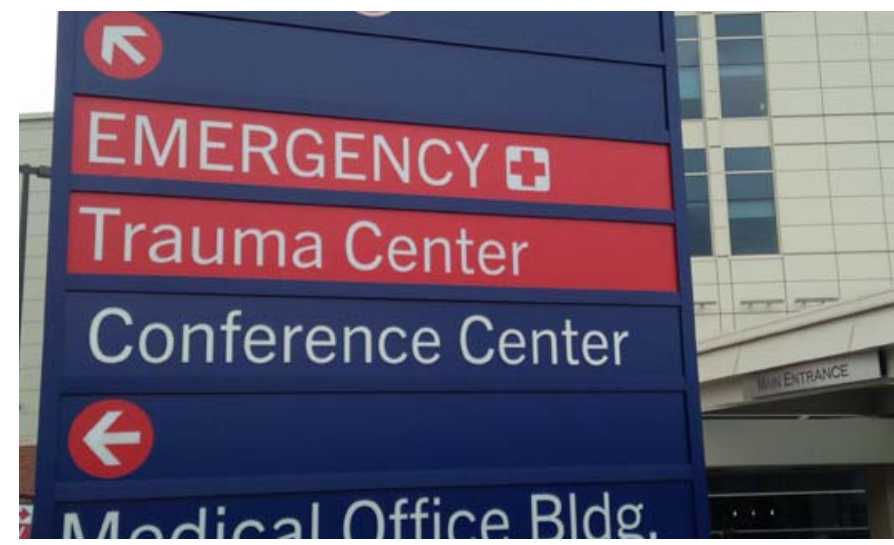

Publication Note:

While the subject matter of this article is not typical to Reconstructive Review, we feel the topic is extremely important given the current COVID-19 health crisis.

\section{Defining Trauma Center Levels}

Trauma centers around the United States are established to provide care to individuals who have been injured and may possibly be in critical condition [1]. Trauma centers are divided into five levels, Levels I-V, with Level I centers fulfilling the most stringent standards and Level $\mathrm{V}$ centers adhering to the least stringent standards. Level IV and V centers are located primarily in rural and underserved areas and are tasked with providing 24-hour emergency care and providing resuscitation. These less comprehensive centers are seen as supplementary care facilities that transfer most patients to a trauma center with a higher designation [1] .

Level III centers are capable of providing continuous surgical coverage with surgeons responding to patient arrival within 30 minutes at most. Level III trauma centers are able to manage most injuries, but they also have to 
transfer many patients to a Level II or Level I center [1] .

Level II trauma centers place a significant emphasis on the delivery of care by an attending surgeon, and they have around-the-clock availability of an attending surgeon. Although residents are permitted to resuscitate patients when an attending surgeon is not immediately present, they cannot deliver care as an equivalent substitute for an attending surgeon. Level II trauma centers receive severely injured patients whose care cannot be comprehensively managed at a trauma center at any of the lower levels. In areas with low population densities, Level II centers serve as the main hospital facilities as Level I centers are geographically distant from these areas. Conversely, in areas with high population densities, Level II centers supplement Level I centers to help provide further resources to care for all severely injured individuals [1].

Level I trauma centers have the exact same standards for quality of clinical care delivery as Level II centers. The two designations are distinguished from one another by additional requirements placed on Level I centers. The criteria for a Level I center includes admitting 1,200 trauma patients annually or admitting 240 patients who have an Injury Severity Score greater than 15, actively conducting trauma research, leading resident training and community outreach programs, and running a critical care service that is surgically directed [1].

\section{Disparities in Trauma Care Among Rural Residents}

Access to trauma centers is a major inequity across the United States. Compared to trauma patients living in states with more clustered centers, trauma patients living in areas where centers are more sparsely distributed experience significantly higher mortality rates [르. Given that the leading cause of death among individuals under the age of 46 is traumatic injury, addressing the geographic disparity in access to trauma centers is a public health issue of paramount importance [ $\underline{3}]$.

As of the most recent 2010 United States Census, approximately 29.7 million Americans lack adequate trauma care, living more than one hour away from a Level I or Level II trauma center by car or helicopter [4]. Living in large cities, suburbs, or high-income areas is associated with better access to trauma care whereas living in rural areas, areas with high uninsurance rates, and areas with high rates of Medicare and Medicaid beneficiaries are associated with poorer access to trauma care [4]. Furthermore, individuals from rural areas are 14\% more likely to die following traumatic injury than non-rural residents, and this disparity is especially prominent at Level I, Level II, and Level IV trauma centers []ㅡ. Increased mortality in rural residents is likely due to a high time to treatment caused by living far away from trauma centers [్]. Rural residents also face disparities in pre-hospital deaths. In particular, subpar prehospital care, injury prevention initiatives, and trauma center access are implicated in prehospital deaths among rural residents, especially with regard to motor vehicular traffic (MVT) injuries and penetrating injuries []].

\section{Potential Improvements to Rural Trauma Care}

A major issue facing rural communities is access to exceptional trauma care for particularly severe injuries. In rural regions where Level I and Level II trauma centers are especially inaccessible, it is common for patients to be taken to Level III trauma centers for stabilization before being transferred to the closest Level I or Level II center [7]. However, if Level III trauma centers were better developed as part of an organized, regionalized system, the number of transfers made to Level I or Level II trauma centers could potentially be reduced [7]. A reduction in transfers is associated with better allocative efficiency of resources from a holistic standpoint and further benefits to a patient and their family [7]. In fact, patients who are not transferred from a Level III trauma center to a Level I or Level II trauma center do not experience a significant difference in mortality, further suggesting a reduction in transfers would be beneficial to the trauma system [7].

Given that Level II trauma centers serve as main hospital facilities in rural areas located far away from Level I trauma centers, rural health outcomes could be improved by incorporating orthopedic traumatologists into patient care teams at Level II trauma centers []]. Compared to general orthopedic surgeons, orthopedic traumatologists perform 16 of 18 procedures more efficiently, which is defined by completing surgeries in less time and subsequently with fewer labor, supply, and implant costs []]. By integrating orthopedic traumatologists more broadly into surgical teams, Level II trauma centers can better allocate limited resources by reducing costs []]. By becoming more efficient, Level II trauma centers can better fulfill their roles as the main deliverer of comprehensive trauma care in sparsely populated areas and as effective complementary care providers in densely populated areas with Level I trauma centers in the vicinity.

Additionally, trauma systems in rural areas would benefit from formally agreeing to share resources, which would improve allocative efficiency and access to trauma care for patients, especially those from rural areas [9]. By better 
placing medical helicopter bases to be able to comprehensively cover a greater area of land, geographic inequities in access to Level II and Level I trauma centers can be better addressed. These inequities can be more directly addressed by building more Level I and Level II trauma centers in rural areas or better staffing and funding Level III trauma centers already located in rural areas so that they can be upgraded to Level II or Level I designations. On a smaller scale, orthopedic surgeons can also opt to utilize more cost-effective implants and devices to make trauma care more affordable and subsequently more accessible, which is especially relevant in making trauma care available to the underinsured and the uninsured [10]. Furthermore, as of 2010, the National Institutes of Health allocated just $0.02 \%$ of its research budget for traumatic injury research [11]. By advocating for the building of new trauma centers the upgrading of existing trauma centers, striving to secure a larger research budget dedicated to trauma research, and making more fiscally conservative decisions regarding patient treatment and transfers without detracting from the quality of patient care, healthcare professionals, especially orthopedic surgeons, can strive to improve the rural trauma care system.

\section{References}

1. Committee on Trauma - American College of Surgeons. Resources for Optimal Care of the Injured Patient. American College of Surgeons; 2014.

2. Brown JB, Rosengart MR, Billiar TR, Peitzman AB, Sperry JL. Geographic Distribution of Trauma Centers and Injury Related Mortality in the United States. Journal of Trauma and Acute Care Surgery. 2016;80(1):42-50.

3. Rhee P, Joseph B, Pandit V, Aziz H, Vercruysse G, Kulvatunyou N, et al. Increasing Trauma Deaths in the United States. Annals of Surgery. 2014;260(1):13-21.

4. Carr B, Bowman A, Wolff C, Mullen MT, Holena D, Branas CC, et al. Disparities in Access to Trauma Care in the United States: A Population-Based Analysis. Injury. 2017;48(2):332-8.

5. Jarman MP, Castillo RC, Carlini AR, Kodadek LM, Haider AH. Rural risk: geographic disparities in trauma mortality. Surgery. 2016;160(6):1551-9.

6. Jarman MP, Hashmi Z, Zerhouni Y, Udyavar R, Newgard C, Ali S, et al. Quantifying geographic barriers to trauma care: Urban-rural variation in prehospital mortality. Journal of Trauma and Acute Care Surgery. 2019;87(1):173-80.
7. Galanis DJ, Steinemann S, Rosen L, Bronstein AC, Biffl WL. Rural Level III centers in an inclusive trauma system reduce the need for interfacility transfer. Journal of Trauma and Acute Care Surgery. 2018;85(4):747-51.

8. Althausen PL, Kauk JR, Shannon S, Lu M, O'Mara TJ, Bray TJ. Operating Room Efficiency: Benefits of an Orthopaedic Traumatologist at a Level II Trauma Center. Journal of Orthopaedic Trauma. 2016;30.

9. Branas CC, MacKenzie EJ, Williams JC, Schwab CW, Teter HM, Flanigan MC, et al. Access to Trauma Centers in the United States . Journal of the American Medical Association. 2005;293(21):2626-33.

10. Genova RC, Box RL, Ficke JR. Accessibility, accountability, affordability: healthcare policy in orthopedic trauma. Current Reviews in Musculoskeletal Medicine. 2017;10(2):258-64.

11. Davis KA, Fabian TC, Cioffi WG. The Toll of Death and Disability From Traumatic Injury in the United States-The "Neglected Disease" of Modern Society, Still Neglected After 50 Years. Journal of the American Medical Association Surgery. 2017;152(3):221-2. 